SHORT REPORT

\title{
Clinical and genetic heterogeneity in peroneal muscular atrophy associated with vocal cord weakness
}

\author{
M McEntagart, M Dunstan, C Bell, E Boltshauser, M Donaghy, P S Harper, N Williams, \\ M D Teare, N Rahman
}

J Neurol Neurosurg Psychiatry 2002;73:762-765

\begin{abstract}
Background: The peroneal muscular atrophy syndrome is the most common inherited disorder of the peripheral nervous system and has extensive clinical and genetic heterogeneity. Cranial nerve involvement is rare, though there are distinct peroneal muscular atrophy syndromes in which vocal cord paralysis is a characteristic feature. Among these $\mathrm{dHMN}-\mathrm{VII}$ and $\mathrm{HMSN}-\mathrm{IIC}$ are clinically similar but are differentiated by sensory involvement in HMSNIIC. The gene for dHMN-VII, designated DHMNVP, has been localised to chromosome 2 q14, but the location of the gene for HMSN-IIC is currently unknown. It has been suggested that $\mathrm{dHMN}-\mathrm{VII}$ and HMSN II-C are allelic disorders.

Objective: To assess the contribution of the $\mathrm{dHMN}-\mathrm{VII}$ predisposition gene to peroneal muscular atrophy syndromes associated with vocal cord weakness.

Methods: Linkage analysis of microsatellite markers at chromosome 2 ql4 was undertaken on two families, one affected by HMSN-IIC and a second manifesting vocal cord paralysis and sensorineural deafness in addition to distal muscular atrophy.

Results: Two-point LOD scores at chromosome 2 ql4 markers encompassing the DHMNVP gene were negative in both families.

Conclusions: These results suggest that at least one further gene predisposing to distal muscular weakness in association with vocal cord paralysis is likely to exist, and that $\mathrm{dHMN}-\mathrm{VII}$ and HMSN-IIC are unlikely to be allelic disorders. Analyses of further HMSN-IIC families are required to confirm this.
\end{abstract}

$\mathrm{T}$ hree groups of peroneal muscular atrophy conditions are currently recognised: a demyelinating form, hereditary motor and sensory neuropathy type I (HMSN-I, or Charcot-Marie-Tooth disease 1 (CMT-1)); an axonal form, HMSN-II or CMT-2; and distal hereditary motor neuronopathy (dHMN, also known as distal spinal muscular atrophy or spinal CMT). ${ }^{1}$ The clinical features of all three forms are broadly similar, as affected individuals present with distal muscle wasting and weakness, variable sensory loss, diminished or absent deep tendon reflexes, and pes cavus deformity of the foot. Molecular studies have shown extensive genetic heterogeneity in this disorder. Currently, at least 15 distinct genetic loci have been mapped and several genes identified. ${ }^{2}$

Cranial nerve involvement is rarely associated with peroneal muscular atrophy. In four dominantly inherited conditions-distal hereditary motor neuronopathy type VII (dHMN-VII), hereditary motor and sensory neuropathy type IIC (HMSN-IIC), scapuloperoneal spinal muscular atrophy (SP-SMA), and vocal cord and pharyngeal weakness with distal myopathy (VCPDM) - vocal cord weakness is characteristic of the disorder.
DHMN-VII typically presents in the second decade and is characterised by distal muscular atrophy and vocal cord paralysis. ${ }^{3}$ Nerve conduction velocities are normal and the EMG shows features of neurogenic atrophy consistent with degeneration of the anterior horn cells. We recently localised the predisposition gene for dHMN-VII to chromosome $2 \mathrm{ql}^{4}$. $^{5}$ The gene was designated DHMNVP (distal hereditary motor neuronopathy with vocal cord paralysis).

HMSN-IIC presents in the second decade and is characterised by weakness of the intercostal and distal limb muscles, vocal cord paresis and by asymptomatic sensory loss. Nerve conduction velocities are normal but sensory nerve amplitudes are reduced. The sensory nerve involvement distinguishes this disorder from dHMN-VII. The location of the HMSN-IIC predisposition gene is currently unknown, but it has been suggested that dHMN-VII and HMSN-IIC may be allelic disorders, caused by mutations of the same gene. ${ }^{6}$

SP-SMA is a neurogenic scapuloperoneal syndrome characterised by congenital absence of muscles, progressive scapuloperoneal atrophy, and vocal cord paralysis. Electrophysiological investigations reveal neurogenic changes with normal sensory nerve studies. ${ }^{7}$ The gene for SP-SMA has been localised to chromosome $12 \mathrm{q} 24 .^{8}$

VCPDM is characterised by vocal cord and pharyngeal weakness associated with a distal myopathy. Electrophysiological studies in affected individuals are not consistent and may show features suggestive of a myopathy or a neuropathy. The gene for this disorder, MPD2, was localised to chromosome $5 \mathrm{q} 31 .{ }^{9}$

HMSN-1 is very occasionally associated with vocal cord weakness. A unique point mutation in gene PMP22 has been reported in a family with HMSN-1, sensorineural deafness, and vocal cord paralysis. ${ }^{10}$ Similar findings were described in one member of a family with a severe demyelinating neuropathy caused by a missense mutation of gene EGR2. ${ }^{11}$ Mutations of PMP22, P0, Connexin 32, and EGR2 genes have also been identified in cases of HMSN-1 and sensorineural deafness or strabismus, but without clinically apparent vocal cord weakness. ${ }^{12} 131415$

To assess whether HMSN-IIC and related disorders may also be caused by the gene predisposing to dHMN-VII we have analysed two families-one affected by HMSN-IIC and a second manifesting vocal cord paralysis and sensorineural deafness in addition to distal muscular atrophy-for linkage to the DHMNVP locus on $2 \mathrm{q} 14$. Both families were also screened for mutations in the PMP22, EGR2, P0, and Connexin32 genes.

Abbreviations: CMT, Charcot-Marie-Tooth disease; dHMN, distal hereditary motor neuronopathy; DHMNVP, distal hereditary motor neuronopathy with vocal cord paralysis; HMSN-I, hereditary motor and sensory neuropathy type I; SP-SMA, scapuloperoneal spinal muscular atrophy; VCPDM, vocal cord and pharyngeal weakness with distal myopathy 
Table 1 Two-point LOD scores at chromosome $2 q 14$ markers spanning the dHMN-VII locus in families D3 and B4

\begin{tabular}{|c|c|c|c|c|c|c|c|c|}
\hline \multirow{2}{*}{$\begin{array}{l}\text { Distance from P-Tel } \\
\text { (cM) }\end{array}$} & \multirow[b]{2}{*}{ Marker } & \multirow[b]{2}{*}{ Family } & \multicolumn{6}{|c|}{ Two-point LOD score at $\theta=$} \\
\hline & & & 0 & 0.01 & 0.05 & 0.10 & 0.20 & 0.30 \\
\hline \multirow[t]{2}{*}{111} & D2S2216 & D3 & -0.00 & -0.00 & -0.00 & -0.00 & -0.00 & -0.00 \\
\hline & & B4 & -0.13 & -0.13 & -0.11 & -0.08 & -0.04 & -0.02 \\
\hline \multirow[t]{2}{*}{115} & D2S274 & D3 & -4.02 & -2.07 & -1.20 & -0.77 & -0.35 & -0.14 \\
\hline & & B4 & -1.02 & -0.88 & -0.57 & -0.38 & -0.17 & -0.07 \\
\hline \multirow[t]{2}{*}{116} & D2S135 & D3 & -4.02 & -2.07 & -1.19 & -0.77 & -0.35 & -0.14 \\
\hline & & B4 & -3.10 & -1.39 & -0.72 & -0.44 & -0.19 & -0.08 \\
\hline \multirow[t]{2}{*}{118} & D2S293 & D3 & -4.02 & -2.07 & -1.19 & -0.77 & -0.35 & -0.14 \\
\hline & & B4 & -0.00 & -0.00 & -0.00 & -0.00 & -0.00 & -0.00 \\
\hline \multirow[t]{2}{*}{122} & D2S 160 & D3 & -0.44 & -0.38 & -0.21 & -0.11 & -0.02 & 0.00 \\
\hline & & B4 & -3.20 & -1.40 & -0.72 & -0.44 & -0.19 & -0.08 \\
\hline \multirow[t]{2}{*}{128} & D2S 100 & D3 & -0.00 & -0.00 & -0.00 & -0.00 & -0.00 & -0.00 \\
\hline & & B4 & 0.00 & 0.00 & 0.00 & 0.00 & 0.00 & 0.00 \\
\hline \multirow[t]{2}{*}{132} & D2S275 & D3 & -0.44 & -0.38 & -0.21 & -0.11 & -0.02 & 0.00 \\
\hline & & B4 & -3.14 & -1.39 & -0.72 & -0.44 & -0.19 & -0.08 \\
\hline \multirow[t]{2}{*}{133} & D2S95 & D3 & -0.44 & -0.38 & -0.21 & -0.11 & -0.02 & 0.00 \\
\hline & & B4 & -3.20 & -1.40 & -0.72 & -0.44 & -0.19 & -0.08 \\
\hline \multirow[t]{2}{*}{141} & D2S 112 & D3 & -1.74 & -1.59 & -1.10 & -0.74 & -0.34 & -0.13 \\
\hline & & B4 & -3.09 & -1.39 & -0.72 & -0.44 & -0.19 & -0.08 \\
\hline \multirow[t]{2}{*}{145} & D2S1334 & D3 & 0.00 & 0.00 & 0.00 & 0.00 & 0.00 & 0.00 \\
\hline & & B4 & -3.09 & -1.39 & -0.72 & -0.44 & -0.19 & -0.08 \\
\hline
\end{tabular}

\section{METHODS}

Two families were selected for evaluation on the basis of phenotypic similarity to dHMN-VII. The clinical features of both these families have been reported previously. ${ }^{16}{ }^{17}$ Permission for the study was obtained from the Bro Taf Health Authority local research ethics committee.

\section{Genotyping and linkage analyses}

DNA was isolated from blood by standard techniques. Ten polymorphic fluorescently labelled microsatellite markers spanning the $20 \mathrm{cM}$ interval harbouring the DHMNVP gene at $2 \mathrm{q} 14^{5}$ were amplified by polymerase chain reaction using standard techniques. The order and distance between the markers is shown in table 1. Amplified markers were electrophoresed on an ABI 377 DNA sequencer and were analysed with GENESCAN and GENOTYPER software (PE Applied Biosystems).

Two-point LOD scores were calculated using the MLINK program of LINKAGE.

\section{Mutation analysis of PMP22, P0, Connexin 32, and EGR2}

P0, PMP22, Connexin32, and EGR2 genes were amplified using standard protocols and published primers. ${ }^{18} 192021$ Mutation screening was undertaken using the WAVE DNA fragment analysis system (Transgenomic).

\section{RESULTS}

\section{Family D3}

Details are given in fig 1A. All individuals had a neurological and electrophysiological examination. The proband II: 1 presented at 43 years with a four year history of diminished manual dexterity and difficulty in walking and a two year history of dysphonia. Examination showed wasting of the small muscles of the hands and of the muscles of the lower legs, and a pes cavus deformity of both feet. A left lateral rectus palsy was noted and laryngoscopic examination revealed left vocal cord paralysis. Generalised areflexia and loss of vibration sense was detected in the legs of the proband's mother I:2 and his older sister II:4. Both were asymptomatic, but distal muscle weakness was elicited on examination. All three showed evidence of an axonal polyneuropathy on electrophysiological testing. Conduction velocities were normal while compound motor action potentials and sensory nerve action potentials were reduced in amplitude. A diagnosis of HMSN-IIC with variable penetrance for vocal cord involvement was made. ${ }^{16}$

\section{Family B4}

Details are given in fig 1B. The proband III: 1 presented at 13 years of age with an unusual gait and impaired exercise tolerance. Examination revealed distal muscle wasting and weakness, areflexia, scapular winging, and thoracic scoliosis. Stridor had been present from six months of age and resulted in a unilateral arytenoidectomy at 10 years. The proband's mother (II:2), maternal grandfather (I:1), and half brother (III:3) gave a history of wasting and weakness of the distal limb musculature from childhood. All three had hearing impairment and I:1 and III:3 had dysphonia. Laryngoscopic examination in I:1, II:2, and III:3 showed unilateral vocal cord paralysis. Electrophysiological testing revealed normal nerve conduction velocities with sensory nerve amplitudes at the lower limit of normal. A diagnosis of distal hereditary motor neuronopathy with vocal cord paralysis and sensorineural deafness was suggested. Neurological, audiological, and laryngoscopic examinations in II: 1 and III:2 were normal. ${ }^{17}$

\section{Linkage analyses}

Family D3

Both affected offspring (II:1 and II:4) share a common maternal haplotype spanning the distal half $(10 \mathrm{cM})$ of the DHMNVP region between D2S160 and D2S1334 (fig 1A). However, II: 1 and his unaffected twin brother II:2 have inherited identical chromosome 2 homologues from their parents. These findings suggest that HMSN-IIC in this family is not linked to chromosome 2q14. Although non-penetrance in II:2 is a possibility, clinical and electrophysiological studies were normal. Two-point LOD scores generated between the disease segregating in this family and markers spanning the DHMNVP locus were negative (table 1 ). The results suggest it is most likely that the predisposition gene in this family is not DHMNVP.

\section{Family B4}

The two affected offspring of II:2 have inherited opposite chromosome $2 \mathrm{q} 14$ homologues from their affected mother, thus excluding $2 \mathrm{q} 14$ as the location of the disease predisposition gene in this family (fig 1B). Negative two-point LOD scores were generated for all markers analysed (table 1). 
A

Family D3
B

Family B4
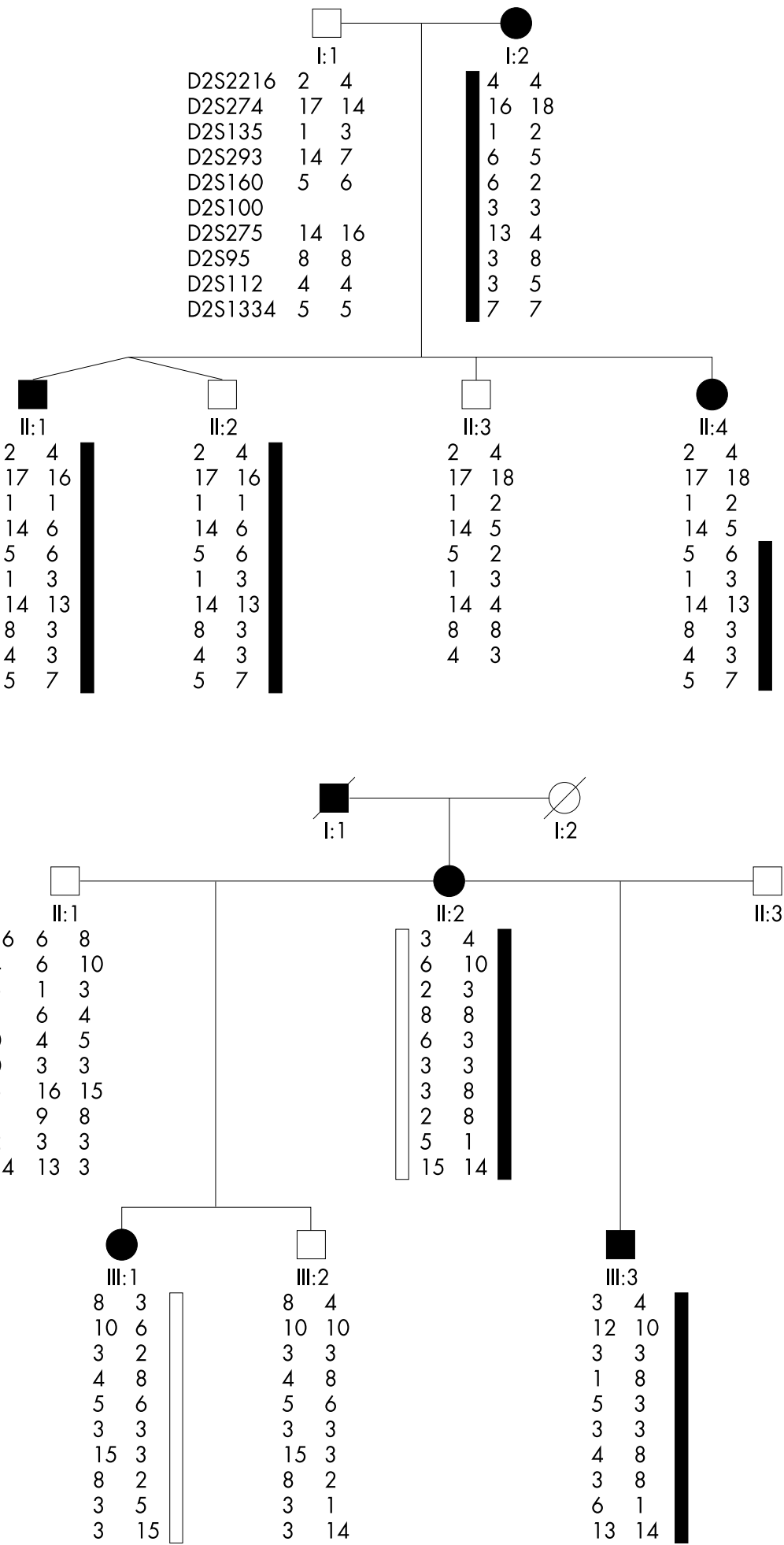

Figure 1 (A) Family D3. (B) Family B4.

No disease causing alteration was identified on mutation screening of PMP22, P0, Connexin32, or EGR2 genes in either family.

\section{DISCUSSION}

We recently mapped the gene for dHMN-VII to chromosome 2 q14 by genome-wide linkage analysis. ${ }^{5}$ We have now examined two families with phenotypes similar to dHMN-VII for linkage to the DHMNVP gene.
Family D3 has HMSN-IIC. HMSN-IIC is clinically similar to dHMN-VII but may be distinguished by the presence of sensory involvement in HMSN-IIC. It has been suggested that HMSN-IIC and dHMN-VII are caused by mutations of the same gene. ${ }^{6}$ There is a precedent for allelism between a form of dHMN and HMSN-II, as dHMN-V and HMSN-IID both map to the same region of chromosome $7 \mathrm{pl} 14 .{ }^{22} 2324$

Our results show that the involvement of family D3 is very unlikely to be caused by mutation of DHMNVP, suggesting 
that HMSN II-C and dHMN-VII are not allelic disorders, and that the HMSN-IIC locus is not located at chromosome $2 \mathrm{ql} 4$. Analyses of additional HMSN-IIC families are required to confirm this.

Family B4 has distal muscular atrophy, vocal cord weakness, and sensorineural deafness. It is convincingly unlinked to the DHMNVP locus on chromosome $2 \mathrm{q} 14$.

Neither family had features suggestive of either SP-SMA or VCPDM, both of which can also be associated with vocal cord weakness. These disorders are distinguished by the presence of scapular muscle wasting in SP-SMA, and by dysphagia and myopathic changes on muscle biopsy in VCPDM. Mutations in the PMP22, EGR2, Connexin32, and P0 genes (which rarely result in peroneal muscular atrophy and cranial nerve involvement) were also excluded in both families.

Our results strongly suggest that the predisposition gene in these two families is not one of those currently known to confer susceptibility to peroneal muscular atrophy in association with cranial nerve weakness. Furthermore, the phenotypes of the two families are different, suggesting the clinical presentation may be caused by mutations of separate genes.

None of the genes for conditions in which vocal cord weakness is characteristic (dHMN-VII, VCPDM, SP-SMA, HMSN II-C) has been isolated, and it is thus difficult to understand why there is preferential involvement of the laryngeal nerves in these disorders. The gene for HMSN-IIC has not been mapped and our results suggest that at least one (and possibly two) further genes predisposing to distal muscular weakness in association with vocal cord paralysis are likely to exist. Identification of these genes will obviously improve our understanding of the pathogenesis of peroneal muscular atrophy. However, they may also provide insight into the development, function, and pathology of vocal cord innervation.

\section{ACKNOWLEDGEMENTS}

We are grateful to all the family members for their kind cooperation in this research. This work was supported by the Muscular Dystrophy Campaign, UK.

\section{Authors' affiliations}

M McEntagart, M Dunstan, P S Harper, N Rahman, Institute of Medical Genetics, University Hospital of Wales, Cardiff; UK

C Bell, Department of Medical Genetics, Medical School, Foresterhill, Aberdeen, UK

E Boltshauser, Department of Paediatrics, University of Zurich, Zurich, Switzerland

M Donaghy, University Department of Clinical Neurology, Radcliffe Infirmary, Oxford, UK

N Williams, Department of Psychological Medicine, University of Wales College of Medicine, Cardiff; UK

M D Teare, Mathematical Modelling and Genetic Epidemiology Group,

Division of Genomic Medicine, University of Sheffield, Sheffield, UK

Competing interests: none declared

Correspondence to: $\operatorname{Dr} M$ McEntagart, Department of Clinical Genetics, St George's Hospital Medical School, Cranmer Terrace, London SW17 ORE, UK; m.mcentagar!@sghms.ac.uk

Received 23 January 2002

In revised form 14 August 2002

Accepted 15 August 2002

\section{REFERENCES}

1 De Jonghe $\mathbf{P}$, Timmerman V, Van Broeckhoven C, and workshop participants of 2nd Workshop of the European CMT Consortium. 53rd ENMC International Workshop on classification and diagnostic guidelines for Charcot-Marie-Tooth type 2 (CMT2-HMSN-II) and distal hereditary motor neuropathy (distal HMN-spinal CMT), September 26-28, 1997, Naarden, The Netherlands. Neuromusc Disord 1998;8:426-31.

2 Vance JM. The many faces of Charcot-Marie-Tooth disease. Arch Neurol 2000;57: 638-40

3 Young ID, Harper PS. Hereditary distal spinal muscular atrophy with vocal cord paralysis. J Neurol Neurosurg Psychiatry 1980;43:413-18.

4 Pridmore C, Baraitser $M$, Brett EM, et al. Distal spinal muscular atrophy with vocal cord paralysis. J Med Genet 1992;29:197-9.

5 McEntagart M, Norton N, Williams $\mathrm{H}$, et al. Localisation of the gene for distal hereditary motor neuronopathy-VIII to chromosome 2ql 4 Am J Hum Genet 2001;68:1270-6.

6 Dyck JP, Litchy WJ, Minnerath S, et al. Hereditary motor and sensory neuropathy with diaphragm and vocal cord paresis. Ann Neurol 1994:35:608-15.

7 DeLong R, Siddique T. A large New England kindred with autosomal dominant neurogenic scapuloperoneal amyotrophy with unique features. Arch Neurol 1992;49:905-8.

8 Isozumi K, DeLong R, Kaplan J, et al. Linkage of scapuloperoneal spinal muscular atrophy to chromosome 12q24.1-q24.31 Hum Mol Genet 1996:5:1377-82.

9 Feit H, Silbergleit A, Schneider LB, et al. Vocal cord and pharyngeal weakness with autosomal dominant distal myopathy: clinical description and gene localization to 5q31. Am J Hum Genet 1998;63:1732-42.

10 Kovach MJ, Lin JP, Boyadjiev S, et al. A unique point mutation in the PMP22 gene is associated with Charcot-Marie-Tooth disease and deafness. Am J Hum Genet 1999;64:1580-93.

11 Pareyson D, Taroni F, Botti S, et al. Cranial nerve involvement in CMT disease type I due to early growth response 2 gene mutation. Neurology 2000;54:1696-8.

12 Tyson J, Ellis D, Fairbrother U, et al. Hereditary demyelinating neuropathy of infancy: a genetically complex syndrome. Brain 1997; 120:47-63

13 De Jonghe P, Timmerman V, Ceuterick C, et al. The Thr 124Met mutation in the peripheral myelin protein zero (MPZ) gene is associated with a clinically distinct Charcot-Marie-Tooth phenotype. Brain 1999; 122:281-90.

14 Stojkovic T, Latour P, Vandenberghe A, et al. Sensorineural deafness in X-linked Charcot-Marie-Tooth disease with connexin 32 mutation (R142Q). Neurology 1999;52:1010-14.

15 Latour P, Gatignol A, Boutrand L, et al. A R381H mutation in the EGR2 gene associated with a severe peripheral neuropathy with hypotonia. J Periph Nerv Syst 1999;4:293-4.

16 Donaghy $M$, Kennett R. Varying occurrence of vocal cord paralysis in a family with autosomal dominant hereditary motor and sensory neuropathy. J Neurol 1999;246:552-5.

17 Boltshauser E, Lang W, Spillman T, et al. Hereditary distal muscular atrophy with vocal cord paralysis and sensorineural deafness: a dominant form of spinal muscular atrophy? J Med Genet 1989;26:105-8.

18 Nelis E, Timmerman V, De Jonghe $P$, et al. Rapid screening of myelin genes in CMT1 patients by SSCP analysis: identification of new mutations and polymorphisms in the PO gene. Hum Genet 1994; 94: 653-7

19 Bergoffen J, Scherer SS, Wang S, et al. Connexin mutations in X-linked Charcot-Marie-Tooth disease. Science 1993;262:2039-42.

20 Timmerman V, De Jonghe $P$, Ceuterick $C$, et al. Novel missense mutation in the early growth response 2 gene associated with Dejerine-Sottas syndrome phenotype. Neurology 1999:52:1827-32.

21 Roa BB, Carcia CA, Kulpa DA, et al. Charcot-Marie-Tooth disease type $1 \mathrm{~A}$. Association with a spontaneous point mutation in the PMP22 gene. N Engl J Med 1993;329:96-101.

22 Christodoulou K, Kyriakides T, Hristova AH, et al. Mapping of a distal form of spinal muscular atrophy with upper limb predominance to chromosome 7p. Hum Mol Genet 1995;9: 1629-32.

23 Ionasescu V, Searby C, Sheffield VC, et al. Autosomal dominant Charcot-Marie-Tooth axonal neuropathy mapped on chromosome $7 p$ (CMT2D). Hum Mol Genet 1996;5:1373-5.

24 Sambuughin N, Sivakumar K, Selenge B, et al. Autosomal dominant distal spinal muscular atrophy type $\mathrm{V}$ (dSMA-V) and Charcot-Marie-Tooth disease type 2D (CMT2D) segregate within a single large kindred and map to a refined region on chromosome 7p 15. J Neurol Sci 1998; 161:23-8. 